https://doi.org/10.36105/iut.2019n29.04

\title{
DERECHO COLABORATIVO. EL CONOCIMIENTO QUE TODO ABOGADO DEBE ADQUIRIR PARA LOGRAR UNA LABOR MÁS EFICIENTE
}

\section{COLLABORATIVE LAW. THE KNOWLEDGE THAT EVERY LAWYER SHOULD ACQUIRE TO ACHIEVE A MORE EFFICIENT JOB}

\author{
Rafael Horacio Montoya Vargas* \\ Centro Estatal de Justicia Alternativa del \\ Estado de Guanajuato, México \\ rafael.montoya@poderjudicial-gto.gob.mx
}

\section{RESUMEN:}

En la actualidad, el jurista necesita formarse, no sólo en los esquemas adversariales, sino, también, en las visiones de cooperación y fomento a la cultura de la paz, que no pretenden relegar la perspectiva personal del conflicto, sino generar un comportamiento colaborativo, responsable y constructivo, que permita a las partes asumir sus obligaciones con base en los intereses recíprocos, a fin de proponer soluciones que puedan ser integradoras y participativas. Este enfoque, que pretende desafiar los paradigmas existentes, exige modificar el plan de estudios de la licenciatura en derecho para dar cabida a una nueva asignatura: el derecho colaborativo.

\section{Palabras clave:}

Práctica colaborativa, gestión del conflicto, escuelas de negociación, cultura de la paz, ganar-ganar.

\section{ABSTRACT}

At present, the jurist needs to be trained not only in the adversarial schemes, but also in the visions of cooperation and promotion of the culture of peace, which do not seek to relegate the personal perspective of the conflict, but to generate collaborative, responsible and constructive behavior, which allows the parties to assume their obligations on the basis of mutual interests, in order to propose solutions that can be participatory and participatory. This approach, which aims

Director del Centro Estatal de Justicia Alternativa del Estado de Guanajuato. 
to challenge existing paradigms, requires modifying the undergraduate curriculum to accommodate a new subject: collaborative law.

\section{Keywords:}

Collaborative law, conflict management, bargaining school, peace culture, win-win.

\section{INTRODUCCIÓN}

Desde el origen más remoto de las civilizaciones humanas una de las principales preocupaciones ha sido la atención y solución de los conflictos suscitados entre sus miembros. Las sociedades primitivas consideraban que la mejor manera de atender las controversias era a través de reglas de observancia general, que constituían verdaderos derechos y obligaciones para cada uno de los miembros de la comunidad.

Cuando las sociedades antiguas comenzaron a estructurarse y organizarse propicia que su sistema de reglas fuera cada vez más complejo y que naciera formalmente lo que actualmente conocemos como ciencia del derecho, para cuyo entendimiento y aplicación eran requeridos operadores expertos.

Los albores de la disciplina se remontan a la antigua Grecia, lugar en donde se representaba por la Diosa Themis, protectora de todos los profesionales que realizaban funciones de abogados o juristas. En ese tiempo fue mucho el prestigio que fue ganando todo aquel que realizara la labor de la abogacía: "Aquel que hace por otro". Nace así una profesión que en su desarrollo se mantendrá siempre ligada a la historia de la humanidad, de la justicia y de los ideales del Estado, los que han sido pilares fundamentales para su desarrollo y evolución permanente, hasta convertirse en una de las profesiones elegidas de manera preferente por jóvenes que culminan sus estudios de bachillerato.

\section{NECESIDAD DE LA CIENCIA JURÍDICA}

La actividad profesional jurídica surge entonces por la imperiosa necesidad de contar, dentro del grupo social, con expertos en la interpretación de las normas emanadas de los Estados debidamente estructurados. Los gobernantes en turno tomaban el cúmulo de reglas que debía observar el ciudadano, tanto de las costumbres sociales como de la moral de los pueblos. Además, los órganos o autoridades que auxiliaban al soberano en la tarea de gobernar creaban leyes sumamente complejas, cuya comprensión e implementación quedaba sólo al alcance de quien dedicaba todo su tiempo al estudio a fin de comprender la instrumentación $\mathrm{y}$, como consecuencia, contar con aptitud de brindar asesoría o servicio legal a quien por cualquier circunstancia quebrantara la paz y el orden.

Las civilizaciones que marcaron el rumbo de los Estados debidamente organizados fueron la griega y la ateniense. Con su pensamiento filosófico y epistemológico determinaron, también, el desarrollo de la justicia. En Atenas se funda la primera escuela de juristas y, según narra la historia, Pericles se convierte en el primer profesional en ejercicio de la función de abogado; sin embargo, no fue sino hasta la aparición de la civilización romana cuando el derecho alcanza su 
punto más alto de desarrollo. Las instituciones de ésta preveían dos figuras estrechamente relacionadas al sistema jurídico; el advocati o causadici que corresponde a lo que hoy en día conocemos como abogados y los jurisconsulti o iurisprudenti que no tenían títulos como los advocati, pero que asistían y auxiliaban a otros en los juicios públicos, además de aconsejarlos para su defensa debida.

Durante los casi tres siglos comprendidos entre la fundación de Roma, año 753 a.C., y hasta la aparición de la compilación Justiniana y la caída del Imperio Romano de Occidente, en 476, el imperio había conquistado gran parte de lo que hoy es Europa, África y algunos territorios de Asia, en los que permeó su sistema jurídico y propició la evolución a costa de las circunstancias que se presentaban en cada uno de los territorios (Morineau, 2006); la ambición expansionista romana los llevó a que, desde mediados del siglo II a.C., sometieran a la península ibérica a un fuerte proceso de romanización, el cual vino a culminar en el año 209 a.C. con la conquista de Nova Carthago (hoy Cartagena) por el general Publio Cornelio Escipión, quien posteriormente fundó la colonia Itálica cerca de Sevilla. La ocupación romana en el territorio español trajo condigo grandes avances en el tema del derecho municipal. A pesar de la fuerte organización española, a la caída del imperio fue perdiendo cohesión y poder, lo que provocó que el pueblo germano de los godos lo invadiera y ocuparan.

El territorio español, ahora dominado por los visigodos (godos del este), fue conquistado intelectualmente por las instituciones, las creencias, las costumbres, los usos y el sistema jurídico existente en el territorio, los cuales conservaron la estructura municipal, en gran medida. En éste, la adopción de la normativa romana, a la cual introdujeron nuevas formas e instituciones, dio origen a la ley romana de los visigodos. Sin embargo, el año 711 marca el inicio de la conquista de los árabes, extendida por toda la península, con excepción de las regiones montañosas de Cantabria y los Pirineos. El dominio durará cerca de ocho siglos, y no será hasta que el rey Pelayo, en Asturias, emprende la reconquista de los territorios invadidos cuando culmina la desocupación, en 1492, con la toma de Granada. Ello propiciará la unificación del derecho (Valencia, 2016).

Con la conquista, los españoles migraron a la Nueva España y con ellos la organización, las instituciones, el derecho y la tradición romano-canónica. En ésta, por impulso de Fray Juan de Zumárraga y de Antonio de Mendoza, primer virrey de la Nueva España, mediante cédula del príncipe Felipe, el 25 de enero de 1553 fue fundada la Real y Pontificia Universidad de México (Universidad Pontifica de México, 2015) que impartía la cátedra de leyes. Para cursarla, los estudiantes debían haber concluido la escuela de letras y gramática latina, esta última tras realizar estudios a lo largo de tres años. Una vez concluidos los estudios universitarios, el alumno podía optar por el grado de bachiller en leyes, que alcanzaba mediante la aplicación de un examen de conocimientos, o de licenciado o doctor en leyes con la presentación y defensa de una tesis.

\section{LA ENSEÑANZA DEL DERECHO EN MÉXICO}

La enseñanza de la disciplina jurídica —en México - la podemos clasificar en dos grandes periodos. El primero comprende desde el virreinato hasta la primera 
mitad del siglo XIX, donde predominó una configuración curricular orientada a la formación académica. El segundo va desde la creación de la Escuela Nacional de Jurisprudencia, en 1867, hasta época actual. En fecha posterior se funda el Ilustre y Real Colegio de Abogados de México (1760) gracias a la cédula emitida por el Rey Carlos III, que tenía como finalidad el ejercicio del mutualismo y ciertos actos de piedad, además de elevar el nivel de la práctica jurídica; de ahí que únicamente aquellos que estuvieran matriculados podrían fungir como litigantes en las audiencias de la Corte de México (Ilustre y Nacional Colegio de Abogados de México, 2018). En 1867, por Decreto de la Ley Orgánica de la Instrucción Pública para el Distrito Federal y Territorios se crea la Escuela Nacional de Jurisprudencia que preparaba, no sólo a los profesionales del derecho, sino también a los escribanos, agentes de negocios y licenciados en economía, la que para 1951 se convertiría en la actual Facultad de Derecho de la Universidad Nacional Autónoma de México (México, y otros, 2013). Y en 1912 se funda la Escuela Libre de Derecho como la primera institución particular de educación superior especializada en enseñar la ciencia jurídica en el país.

Con el devenir de los años, la profesión de abogado no sólo se fue consolidando, sino que, además, se convertirá en la profesión favorita de quienes dirigirán los destinos políticos de la Nación, tal vez basados en el primer reto que cualquier gobernante enfrenta en su gestión: consolidar un verdadero Estado de derecho. Por la cabeza de cualquier gobernante, primero deben sentarse las bases para que los gobernados perciban la seguridad jurídica que el Estado les brinda.

La dignidad y popularidad que representaba tener un título de abogado, que además repercutía en el estatus social y económico de quien ejercía tan noble profesión, como consecuencia devino la saturación de la licenciatura y, por lo menos en México, cada vez surgían más escuelas o facultades de leyes. Algunas incluso con el ofrecimiento de que el experto en dicha disciplina lograría su culminación en tan solo dos años, lo que dejaba mucho que desear sobre el nivel académico de dichas instituciones. No obstante, el crecimiento y la evolución, el currículo de casi todas las escuelas o facultades de derecho mantenían los programas tradicionales en donde las materias básicas como derecho romano, derecho civil, derecho penal, derecho agrario, derecho mercantil, y los respectivos procesales, eran las materias "estrellas" de los programas de estudio.

\section{NECESIDAD DE NUEVAS ASIGNATURAS}

No será hasta hace aproximadamente diez años, cuando se empiezan a incorporar nuevas asignaturas jurídicas en los planes de estudios respectivos; algunas pasajeras, pero otras llegaron para quedarse, como el derecho ecológico y una que de modo particular reviste importancia para este ensayo; la materia denominada mecanismos o métodos alternos o alternativos de solución de controversias o conflictos, conocida por el acrónimo: Masc. Surge, así, el estudio de las soluciones alternas de controversias que, en algunas instituciones educativas, los alumnos la han cursado por elección, dado que es de carácter optativo. Aun así, estimamos que representa un avance de consideración, que, al estudioso de la 
disciplina jurídica, a lo largo de sus estudios le muestren un paradigma distinto al de la justicia adversarial. No obstante, para reforzarlo hacen falta otras asignaturas bajo este enfoque.

\section{DERECHO COLABORATIVO}

La cuestión de si la materia debe conformar o no el currículo obligatorio; cuál debe ser el contenido del programa de estudios respectivo, o incluso su denominación precisa, aunado a la carencia de textos que apoyen su compresión, han impedido que el nuevo paradigma detone acorde con las necesidades actuales de búsqueda de otras salidas al conflicto. Por tanto, nos parece lógico y comprensible definir una nueva rama de la ciencia jurídica que favorezca el estudio homogéneo y efectivo - aun a manera de introducción o antecedente- de los mecanismos alternos de solución de controversias; incluso desde un enfoque jurídico, como les gusta a quienes diseñan los programas de estudios de la licenciatura multicitada. A partir de una estructura legal nueva que resulte de comprensión más sencilla para docentes y alumnos, resultaría atractivo ofrecer la materia que denominaremos: derecho colaborativo, en razón de que no los sacará de su esquema conceptual el que, en una licenciatura en abogacía, de necesidad deben estudiarse derechos.

Así, al hablar de derecho colaborativo se continúa bajo el estereotipo de compresión y estudio de la ciencias o materias que conformen el plan de estudios de la carrera de abogado. El derecho colaborativo convendría al gremio educativo. Al momento del desarrollo del programa, pronto se darían cuenta de que lo que se busca es la preparación de los abogados, no para el litigio o la confrontación legal, sino para aprender a construir acuerdos mutuamente satisfactorios y buscar que los clientes se encuentren satisfechos por la asesoría o representación que les fue dada, al apreciar que sus antagonistas, los que no serán más adversarios, también serán vencidos.

Antes de postular una nueva disciplina jurídica resulta indispensable analizar la problemática que, en un momento dado, justifica esta postura. Para ello basta con comparar, por cruda que sea, las deudas que la hetero-composición tiene con la sociedad. Si partimos de la base de que el derecho en el momento actual tiene como característica fundamental la forma hetero-compositiva de resolución de los conflictos, habría que partir del principio básico de que es otro, un tercero ajeno a las partes en conflicto, quien resuelve sus diferencias basado en un sistema de normas, y es ahí precisamente donde encontramos la situación problemática, ¿cuántas veces la resolución dictada por el tercero ajeno al conflicto no ha dejado satisfecha ni a la persona a quién se decretó ganadora del proceso, mucho menos a quien resultó perdedor? Y éste no es el único dilema, sino, además, el grado de satisfacción que, a las partes, deja la intervención del órgano jurisdiccional respecto de la controversia que le plantearon a través de sus escritos procesales.

\section{ANÁLISIS DE UN CASO PRÁCTICO}

En los primeros años de la profesión, después de haber recibido toda la formación adversarial tradicional suficiente para la atención de los conflictos planteados 
por los futuros clientes, tuvimos oportunidad de atender la petición de una señora, quien deseaba asesoría y representación en juicio por la infidelidad sufrida por parte de su esposo, del cual: "Nada quería saber". Su testimonio principal sostenía que, en fecha reciente, se había enterado de que su todavía cónyuge había tenido un hijo fuera de matrimonio, e incluso lo había reconocido. A partir de esta petición, nuestro pensamiento lógico-jurídico, basado en las enseñanzas de confrontación y adversariales de nuestros formadores en la profesión, entendimos o deducimos que lo que la señora quería o pedía era la disolución del vínculo matrimonial con el hombre que había traicionado el juramento de fidelidad mutua que se debe toda pareja, y que el plus en nuestros servicios sería dejarla satisfecha; por supuesto: "dándole con todo al infiel".

La estrategia a seguir fue la siguiente: la demanda de divorcio fue interpuesta y en la misma fue invocada, como causal, el adulterio; bajo la manga se tenía un as para su procedencia, el acta de nacimiento donde el esposo reconocía haber tenido un hijo con otra durante el tiempo en que había estado casado con la defendida. El hombre casado no puede o, mejor dicho, no debe, reconocer un hijo procreado con mujer distinta de su esposa. De forma simultánea fue presentada una denuncia ante el ministerio público por el delito de incumplimiento de las obligaciones de asistencia familiar. En ésta, como probable responsable fue señalado el esposo adúltero, pues con motivo de la actividad extramarital desatendía las necesidades básicas de su familia. Ambos escritos fructificaron, y al poco tiempo fue emplazado en el juzgado civil (en ese entonces no existían los juzgados de familia en el Estado de Guanajuato) y después de haber dado contestación, los agentes ministeriales cumplimentaron la orden de aprehensión otorgada por el juez: el varón fue remitido a uno de los centros de readaptación social del Estado.

Después del pago de la fianza respectiva, el demandado nos buscó como abogados de la contraparte, y ofreció allanarse a las pretensiones planteadas en la demanda civil. Insistió en que se convenciera, a su todavía esposa, para que le otorgara el perdón en el proceso penal respectivo, pues éste se sigue a petición de parte agraviada, no de oficio. La oferta resultaba tentadora; una vez que recibió y firmó el escrito de allanamiento, la clienta hizo lo propio en el juzgado penal. Como consecuencia, la sentencia en el proceso civil fue favorable. Como abogado, la satisfacción fue plena, en razón de que habíamos llevado a cabo una excelente representación jurídica, pues, en la práctica, la imperiosa necesidad de justicia de quien la solicitó fue concretada: obtuvo el fallo favorable a sus intereses.

No obstante, la historia no termina aquí, sino un año después. Al ir transitando por una de las calles de Guanajuato, nos percatamos de una pareja de enamorados que buscaba pasar inadvertida de nuestra vista, pensamos que se trataba de algún familiar o de alguna persona cercana que por alguna razón esquivaba de nuestras miradas su tórrido romance; con asombro, duda y curiosidad, nos acercamos a la pareja y, nuestra sorpresa fue mayúscula: eran aquellos que meses atrás habían disuelto el vínculo matrimonial con nuestra intervención y apoyo. La clienta había regresado con su exmarido. Les dimos un afectuoso saludo, mismo que devolvieron un tanto apenados, acompañado de una sonrisa. En ese entonces, con la sola formación como abogado adversarial pensamos en la 
máxima popular que afirma que los seres humanos somos el único animal capaz de, "tropezar dos veces con la misma piedra". La situación resultaba simpática, sin hacer mayor reflexión analítica sobre lo peculiar.

Después de siete años de llevado ese asunto y en la época en la que iniciamos el conocimiento particularizado sobre la mediación y la conciliación como procedimientos de autocomposición asistida, la postura adversarial fue cambiando y en el auge de nuestra función como mediador recordamos aquella historia del divorcio de la esposa engañada; entonces sí realizamos un análisis más profundo sobre lo que había pasado años atrás, y de modo más particular sobre si como abogados, dentro del sistema de justicia tradicional habíamos cumplido con la necesidad que en ese momento tenía la esposa engañada, hubiese sido muy enriquecedor poder volver a entrevistar a la clienta y preguntarle, cuáles fueron los motivos que la llevaron a reconciliarse con su ex esposo, y, más aún, si nuestra intervención como abogados le pareció adecuada, no pudimos hacerlo, pero surgieron múltiples reflexiones, las cuales resumimos a continuación.

La primera es que, nuestra labor como abogado en el sistema adversarial, fue no sólo la esperada, sino incluso la apropiada. La segunda va dirigida al juzgador, cuya intervención en razón de lo que pedían y le probamos las partes, seguro que también fue adecuada. Como tercera, tal vez la más devastadora, es si la satisfacción del ideal justicia, como la definiera Justiniano, que consiste en, "dar a cada quien lo que le corresponde", había sido colmada con la intervención de todos los que participamos en aquél juicio y logrado la sentencia respectiva en 1995. Como mediadores, seguro tendríamos que reconocer que, aún dentro del servicio de administración de la justicia, ambas partes no fueron satisfechas en sus necesidades elementales.

Tal vez, la esposa engañada, lo único que quería era la aceptación expresa y tácita de la infidelidad del esposo, así como el ofrecimiento de disculpas y las muestras de un arrepentimiento tal, que le hicieran pensar que nunca más le volvería a fallar. Lo anterior difícilmente podría lograrse con la intervención de alguna autoridad estatal. Exigía, más bien, la intervención de algún tercero que pudiese llevarlos a analizar el conflicto, las necesidades, las responsabilidades y los ánimos para modificar las situaciones que lo produjeron, pero, en ese entonces, en México no existía la mediación y los abogados no estábamos preparados para ver los conflictos jurídicos desde otro enfoque, con una visión basada, más en las necesidades emocionales de las partes, que atendiera a los sentimientos y buscara acuerdos mutuamente satisfactorios. De seguro que el hombre adultero también tenía necesidades insatisfechas y él las podía haber expresado en un procedimiento distinto en el que se le diera voz y participación para encontrar, entre ambos, una solución más atinada a su conflicto de pareja.

Resulta obvio que el hecho de haber regresado como pareja fue un ejemplo claro de que el sistema de justicia formal o heterocompositiva falló en el cumplimiento de las necesidades de solución del conflicto familiar. El análisis de este caso nos muestra que la justicia formal, en ocasiones, le queda a deber a la generalidad en la atención de sus necesidades, que si bien se basa en derechos, también 
involucra cuestiones diversas, como la afectiva, que por su carácter no son cuantificables en numerario ni pueden atenderse por el sistema tradicional de justicia, dada su restricción que entrevé solo lo que la norma predica o dicta, y cuyas respuestas, en algunos casos, generan más problemas que soluciones. Así, podemos afirmar que la sentencia concluye con el juicio o proceso, pero no siempre termina o resuelve el conflicto jurídico-social, o incluso el personal de quienes participaron en un litigio.

No es pertinente hacer un juicio crítico en contra del juzgador o de los abogados, porque nadie tenía obligación de visualizar un esquema distinto para la atención de las necesidades jurídico sociales de las partes en controversias como la narrada, por eso resulta necesario introducirnos en el análisis de una nueva formación del abogado que lo lleve a vislumbrar las preocupaciones de sus clientes y le posibiliten entender mejor sus verdaderas necesidades en torno a la intervención del Estado en la solución de conflictos interpersonales, en las cuales pueda tener participación un tercero sin potestad para obligar, pero dotado de otras herramientas mejores para procurar alcanzar soluciones por las propias partes interesadas, como el mediador o el conciliador.

\section{DE NUEVO A LA CUESTIÓN}

Aun con todo lo expuesto pudiéramos concluir que siguen siendo incipientes los argumentos para establecer la necesidad de creación de una nueva rama del derecho, y mucho menos la autonomía del que hemos denominado: derecho colaborativo, porque para lograrlo necesitamos exponer las razones que motivan la creación, además de resolver las interrogantes del estudio independiente soportado en la doctrina y la legislación sustantiva y adjetiva, pero si faltaren elementos para concebirla, sí debe entenderse como una nueva forma del ejercicio profesional del abogado y, primer paso, debemos trabajar en habilitar a los profesionales del derecho en otras técnicas para llevar acabo el ejercicio profesional, en las cuales favorezcamos la búsqueda de acuerdos para el cliente que, en un primer momento, excluyan el conflicto de la vía judicial, dentro de la justicia formal, a fin de que se colabore con la parte contraria en la búsqueda de acuerdos mutuamente satisfactorios, los que serán legítimos y de observancia puntual, porque surgirán de las propias partes en disputa. Esta nueva concepción jurídica, aunque pareciera novedosa, en realidad tiene como antecedente primario la postura planteada, desde los años setenta, por Frank Sander, matemático y abogado, profesor emérito de la Universidad de Harvard, profesional que sienta las bases de los tribunales multi-puertas, también conocidos como multi-door courthouse: un sistema novedoso en la administración de justicia de acceso para los ciudadanos comunes en los Estados Unidos de América. En éste se exponían las variantes que debe tener toda persona para resolver sus conflictos, de modo semejante a un buffet, donde cada cual elige lo que más le apetece para saciar su apetito, aunque en este caso sería más preciso afirmar: para saciar su sed de justicia. En época más reciente y también en dicho país (noventas) se habla de una 
normativa marco en el desarrollo del derecho colaborativo y los mecanismos alternos (Uniform Law Collaborative Act, 2009).

Otro claro ejemplo de cómo la colaboración no es una de nuestras principales estrategias a seguir en las diferente circunstancias que se nos presentan en nuestra vida, la basamos de modo fundamental en que nuestro desarrollo como persona vamos obteniendo ciertos elementos que nos hacen ser de cierta forma, caracteres que determinan nuestro comportamiento. La forma en la que lo adquirimos es mediante el aprendizaje, desde la enseñanza en la familia dentro de nuestra casa, la escuela e incluso el o los grupos sociales en los que nos desenvolvemos al formar parte de una sociedad. Y es entonces cuando en nuestra interacción con nuestros semejantes se ve reflejada una vez más la característica relativa a la competitividad, y es que precisamente en estos grupos socializadores a los que pertenecemos nos van enseñando consciente o inconscientemente a competir pensando que esta es la única forma de obtener el mayor beneficio.

\section{SI AMBOS JUGADORES SON COLABORATIVOS OBTENDRÁN MAYOR PUNTAJE}

En un ejercicio practicado con grupos de alumnos de la licenciatura en derecho para visualizar qué tipo de negociadores son o serán, los resultados obtenidos fueron más que claros sobre nuestra tendencia natural a competir. La actividad propuesta fue la práctica del juego de cartas denominado: "Serpientes y conejos", que consiste en obtener la mayor cantidad de puntos posibles en cinco tandas de juego. En el juego, todos los competidores, de modo simultáneo, deben tirar una carta en una mesa, sin que puedan ver cuál tirarán los otros, para que al final se vea cuál eligió cada uno. Con las cartas sacadas por cada uno, los resultados que se pueden obtener son en los términos siguientes: si uno de los jugadores juega con la carta de conejo y otro la de serpiente, el segundo obtiene tres puntos, mientras que el primero no obtiene puntos; si ambos jugadores sacan conejo, cada uno obtienen dos puntos, y si ambos sacan serpiente, ganan un punto cada uno.

Una vez explicadas las instrucciones inició el juego y, en la mayoría de los casos, los jugadores que obtenían más puntos optaban por jugar víbora, mientras que los que conseguían menos empezaban jugando conejo, pero, en las últimas tandas también decidieron sacar su carta de víbora. Al preguntarles sobre los motivos que los llevaron a la selección de la carta que tirarían, los que eligieron víbora, en su mayoría aplicaban la lógica de que si en cada turno sacan esta carta iban a ganar o a empatar, pero nunca perderían, y dado que los "perdedores" tiraban conejo, no en todos los turnos, el competidor destacado obtenía siete puntos, dado que él tiraba siempre víbora, pero su contrincante sólo en uno de los casos tiró conejo.

Al finalizar los juegos, los aparentes ganadores estaban muy contentos, porque consideraban que habían triunfado por haber obtenido más puntos; sin embargo, ésta no es la estrategia mediante la cual se obtiene la mayor cantidad de puntos en este juego: si todos hubiesen tirado conejo, ambos jugadores habrían 
obtenido diez puntos, es decir, tres puntos más que aquel que "pensó muy bien" su estrategia de juego.

Esta sencilla actividad reflejó cómo ignoramos el hecho de que si ambos jugadores son colaborativos obtendrían mayor puntaje; éste es el objetivo principal del juego.

\section{LA COLABORACIÓN EN EL DERECHO}

Si trasladamos este juego simple, al derecho, refleja que la colaboración puede ayudarnos a obtener lo que buscamos sin que la parte contraria pierda. Implica un ganar-ganar. Mediante el diálogo puede accederse a las necesidades del otro, además de tener un panorama más completo sobre lo que originó el conflicto; de esta forma cada parte siente que ha sido escuchada y que realmente su postura será tomada en cuenta para dictaminar la resolución favorable para ambas, ya que es flexible a las necesidades de cada uno.

Al incluir una formación colaborativa no competitiva, en la formación de los abogados, se irá inhibiendo el deseo de contender y, poco a poco, lo natural será buscar la cooperación para obtener el mayor beneficio para todos los involucrados. De ahí que estimamos necesaria la implementación del aprendizaje de este tipo en la formación, no sólo del futuro abogado, sino incluso para la vida diaria, con el propósito de dejar de lado el deseo de vencer y llegar a la comprensión de que se puede ganar sin necesidad de que el otro pierda.

Esta práctica colaborativa tiene diferentes ámbitos de aplicación, casi en cualesquiera de los derechos de libre disposición; por ejemplo, resaltan los resultados fructíferos en la atención integral del conflicto en las materias familiar y civil, en donde se tienen registradas experiencias de alto grado de satisfacción entre los conflictuados, que documentan la resolución encontrada de manera colaborativa, lo que permite a las partes explorar el origen, el desarrollo y la cúspide de su conflicto para asumir sus obligaciones de manera responsable basándose fundamentalmente en la exposición de los intereses que avalan las posiciones de cada cual, para que al final propongan soluciones responsables, integradoras y participativas, más allá de beneficiar a uno sólo de ellos.

El derecho colaborativo tiene características que le dan uniformidad; entre otras, la exclusión de la vía judicial como única opción para solucionar las controversias; el diseño u observación de un procedimiento informal que cada parte y sus abogados pueden elegir y variar según su conveniencia, además del elemento final y fundamental: el espíritu colaborativo entre los conflictuados y sus abogados.

En lo referente a los sustentos doctrinales y normativos que pudiesen avalar al derecho colaborativo como nueva disciplina jurídica podemos mencionar los escritos enriquecedores que en nuestro país han presentado los autores siguientes: Ana Ballesteros Temprano, "El derecho colaborativo una forma más humana para la resolución de controversias"; Luis Fernando Saucedo Morales, "Derecho colaborativo una nueva forma de solucionar conflictos", y Antonio M. Prida, "Derecho 
colaborativo otra forma de resolver conflictos", entre otros que ya han empezado a sembrar la inquietud sobre esta nueva rama o conocimiento obligatorio en la formación de los futuros abogados.

En el ámbito normativo se puede rescatar el antecedente de la figura de la transacción que los códigos civiles prevén acerca de la solución buscada por las partes haciéndose recíprocas concesiones, además de las leyes estatales de justicia alternativa, la Ley Nacional de Mecanismos Alternativos de Solución de Controversias en Materia Penal, y, claro como parte fundamental la Constitución Política de los Estados Unidos Mexicanos, en el artículo 17 cuando refiere que, "siempre que no se afecte la igualdad entre las partes, el debido proceso u otros derechos en los juicios o procedimientos seguidos en forma de juicio, las autoridades deberán privilegiar la solución del conflicto sobre los formalismos procedimentales". Y si bien estas nociones pudieran estimarse contradictorias con las ideas expresadas, de que la característica fundamental del derecho colaborativo es la exclusión de la vía judicial, no es tal, pues la norma constitucional pretende alentar las soluciones pacíficas no adversariales, por ende, colaborativas.

\section{LA REGULACIÓN ACTUAL RESULTA INSUFICIENCIA PARA LA ATENCIÓN MEJOR DEL CONFLICTO}

El marco normativo vigente en esta época no regula la rama del derecho que proponemos, por lo que en la Academia será necesario realizar mayores esfuerzos para robustecer la parte doctrinal para que ésta lleve a los órganos legislativos a regular de manera más particularizada el tema y logremos también una postura legislativa sobre tan novedosa rama de la ciencia jurídica.

Otra cuestión que proponemos analizar para sustentar la propuesta del derecho colaborativo es la relación que guarda con los mecanismos alternos de resolución de controversias, en particular con la mediación; la estimamos una mediación sin mediadores. La práctica colaborativa tiene base en dichos mecanismos, pero, por ejemplo, a diferencia de la mediación tendrá un protagonista ajeno a las partes que facilita el proceso, y esta participación podrá recaer en los abogados formados en derecho colaborativo, de tal suerte que ahora ellos serán los auxiliares para que las partes resuelvan su conflicto de manera auto-compositiva y asistida. Lo anterior justificaría que los futuros abogados estudiaran estos temas para convertirse en mejores asesores y representantes de sus clientes. El derecho colaborativo prepararía al futuro abogado en la comprensión del tema derivado de los conflictos jurídicos sociales, para que éste aprendiera a entender las necesidades y el verdadero conflicto que lleva a la persona a su despacho, al tiempo que propiciara mayor entendimiento y empatía entre el profesional y el potencial interesado en busca de asesoría jurídica.

El segundo elemento de estudio sería el análisis de las diversas formas de gestión o solución de los conflictos para que comprenda que éstos no se resuelven sólo de manera hetero-compositiva y adversarial, sino que tiene a su disposición otras formas: auto-tutelares y auto-compositivas. 
Los siguientes temas que acompañarían el aprendizaje del derecho colaborativo tienen que ver con la negociación y las diferentes escuelas que nos enseñan la comprensión del difícil arte de transigir en forma exitosa. Como tema central se abordarían las bondades de los acuerdos negociados y la capacidad que todos tenemos para colaborar desde una perspectiva jurídica, a partir del estudio de la Constitución y de las diferentes figuras procesales que regulan los antecedentes del contrato de transacción, además de las leyes nacional y estatales que abordan los mecanismos alternos de solución de controversias o justicia alternativa. De tal suerte, al finalizar el estudio, el alumno conocerá, explorará y profundizará en el estudio de un nuevo paradigma sobre la gestión de los conflictos, y éste le posibilitará, más tarde, ya como abogado, ofrecer a sus clientes otra modalidad de gestión del mismo, cuando le planteen un asunto para su pronta solución: podrá hablarles de una visión nueva acerca de sus controversias, no la forma tradicional o adversarial de resolverlos, sino de la posibilidad de encontrar una solución colaborativa.

Con el aprendizaje sobre el paradigma colaborativo, la asesoría que podría brindar el Licenciado en Derecho sería más completa, y estaría no solamente favoreciendo más variantes en los esquemas de atención procesal; con mayor significado, también estaría contribuyendo a la cultura de la paz, que tanta falta le hace a la sociedad contemporánea. De pronto, como integrantes de la sociedad comprenderíamos que, no solamente peleando podemos resolver las disputas; que una sentencia no siempre pone fin a un conflicto, sino que, tal vez, dará nacimiento a uno nuevo o de mayor envergadura. Lo que la sentencia termina es el proceso jurisdiccional, pero en muchos casos no concluye la controversia ni el conflicto, mucho menos resuelve las necesidades de los que fueron llevados al litigio adversarial.

Se trata, pues, de un desafío que engloba al estudio tradicional de la ciencia jurídica, y qué, como consecuencia, puede no ser bien visto por los conservadores atados a los esquemas tradicionales ni por aquellos a quienes les gusta continuar haciendo las cosas de la misma manera.

Por tanto, se requiere una visión futurista dispuesta a desafiar los paradigmas existentes para lograr la consolidación de nuevos programas de estudios de la ciencia del derecho en los que tenga cabida el derecho colaborativo, sea que se cuestione o no si merece considerarse una rama autónoma que amerite un estudio independiente de las otras, o de un tema que forme parte de alguna disciplina ya consolidada, pero, lo que desde ya estimamos como verdad innegable, es que el jurista debe formarse no sólo en los esquemas adversariales, sino también, y acaso más aún por las necesidades sociales en las que transita nuestro país, en las visiones de cooperación y de fomento a la cultura de la paz que no pretenden excluir la visión propia del conflicto, sino que buscan generar un comportamiento colaborativo que excluya la visión del conflicto desde el enfoque destructivo y más bien generen una visión constructiva del mismo. 
En definitiva, como soporte y fundamento de nuestra propuesta, tendríamos que tomar en cuenta y valorar la eficacia que han tenido en México — durante los último veinte años - los centros de justicia alternativa de las diversas entidades federativas, que al cabo de estos años de actividad intensa han consolidado su aportación a la cultura de la paz y a la nueva concepción pública que busca resolver los conflictos en forma auto compositiva, la cual tiene como fundamento cardinal la responsabilidad de los generadores y se traduce en una participación más comprometida de quien afronta desavenencias y coopera en la búsqueda de soluciones. Esta participación los convierte en mejores ciudadanos, responsables de sus actos y de las probables consecuencias, pues los asumen con el propósito de enmendar lo negativo que abonó al demérito de los valores de la sociedad mexicana y que en ocasiones incluso menoscabó la dignidad de algunos de sus miembros. 